\title{
SPECTROPHOTOMÉTRIE INTÉGREE DES GALAXIES \\ PROCHES DANS L'ULTRAVIOLET (EXPÉRIENCE PERSÉE)
}

\author{
P. CRUVELLIER, A. ROUSSIN et Y. VALERIO \\ Laboratoire d'Astronomie Spatiale du CNRS, et Observatoire de Marseille, France
}

\begin{abstract}
Preliminary results obtained from a sounding rocket launched in December 1968 show a strong ultraviolet excess in the central part of the Andromeda nebula, as compared to the color of the sky background.

The color index of M 31 in this spectral range may be explained by an abnormally high proportion of B stars.
\end{abstract}

\section{Introduction}

'Persée' est avant tout un spectrophotomètre photoélectrique de précision à 4 bandes passantes simultanées qui a été réalisé grâce à la collaboration du Centre National d'Etudes Spatiales.

La définition de l'instrument est liée essentiellement à la stabilisation; elle est également liée à la précision probable d'acquisition $( \pm 1.5)$. Cela conduit à la nécessité, pour être sûr d'acquérir un astre donné, de concevoir un instrument avec au moins $3^{\circ}$ de champ. Nous avons choisi $4^{\circ}$.

Un spectromètre de $4^{\circ}$ de champ risquait d'avoir bien peu d'intérêt. On a préféré concevoir l'appareil, dont la pupille d'entrée a $15 \mathrm{~cm}$ de diamètre, pour qu'il donne un champ de $1^{\circ}$, mais en effectuant un balayage bidimensionnel sur $16^{\circ}$ carré $\left(4^{\circ} \times 4^{\circ}\right)$. Ainsi, l'acquisition est certaine, et la définition angulaire sur le ciel, permet d'étudier plusieurs astres isolés situés dans le champ, ou bien un astre étendu de grande dimension dont la spectrophotométrie peut être étudiée en 16 régions différentes de $1^{\circ}$ chacunes.

'Persée' a été tirée en décembre 1968 sur la nébuleuse d'Andromède (M 31). Cette expérience avait pour but de préciser le taux des populations d'étoiles de $M 31$ (région centrale) et de mieux définir les limites de cette galaxie par rapport au fond du ciel qui, au sol, sature rapidement à cause de la luminance du ciel nocturne et de la répartition générale d'étoiles galactiques.

\section{Principe de l'Optique}

Le principe du monochromateur du type filtre B. P. M. a été décrit en détail (Bonnet et Courtès, 1962; Courtès, 1962); nous n'y reviendrons pas ici. Rappelons simplement que le monochromateur qui disperse les pupilles au lieu de disperser l'image de l'astre a, grâce à ce dispositif, des bandes passantes indépendantes des défauts de guidage de la plateforme de stabilisation de la fusée (Figure 1) et présente l'avantage d'illuminer d'un éclairement uniforme la photocathode des photomultiplicateurs, sans qu'il soit nécessaire d'adjoindre une lentille de Fabry. 


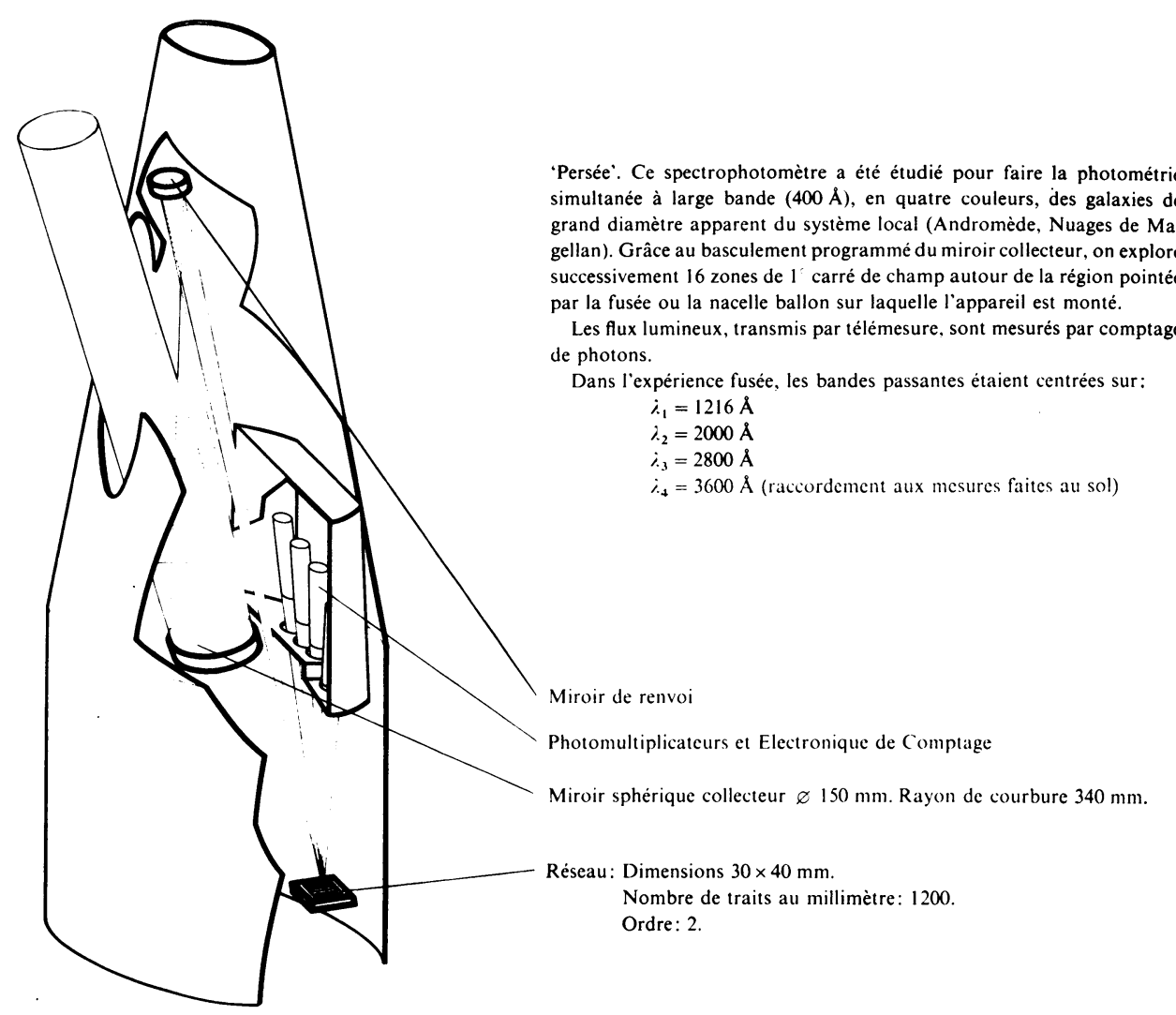

Fig. 1.

Le flux monochromatique est recueilli par le photomultiplicateur travaillant en comptage d'impulsions. L'information est transmise au sol par télémesure.

Les 4 bandes passantes de $400 \AA$ de large, étaient centrées sur: 1216, 2000, 2750, et 3450 A.

La Figure 2 donne la forme de ces bandes passantes.

Avant le tir, des calibrations avaient permis, non seulement de connaître la forme exacte des bandes passantes, mais encore de déterminer la fonction de transfert de l'appareil permettant ainsi l'établissement des rapports absolus des différents éléments spectraux.

\section{Résultats}

Par suite d'une saturation due à la télémesure, seules les deux bandes passantes $(3450 \AA$ et $2750 \AA$ ) ont donné des résultats exploitables.

Dans le domaine $2750 \AA$, les étoiles de la région marginale de la Voie Lactée superposable à la nébuleuse d'Andromède jouent un rôle de moins en moins grand dans la luminance moyenne du fond du ciel (chute de la répartition spectrale énergétique 
des étoiles galactiques). C'est ce que l'on constate sur les résultats de 14 des champs de $1^{\circ}$.

Ainsi, à l'exception des deux carrés correspondant au signal de $\mathrm{M} 31$, le fond du ciel dans la bande $3450 \AA$ présente, d'un degré carré à l'autre, les fluctuations dues aux irrégularités de la répartition des étoiles galactiques. Ces fluctuations disparaissent complètement sur les résultats du fond du ciel $2750 \AA$ ce qui confirme le bien fondé de la recherche des limites extrêmes de M 31 (Figure 3) ou de tout autre système extragalactique.

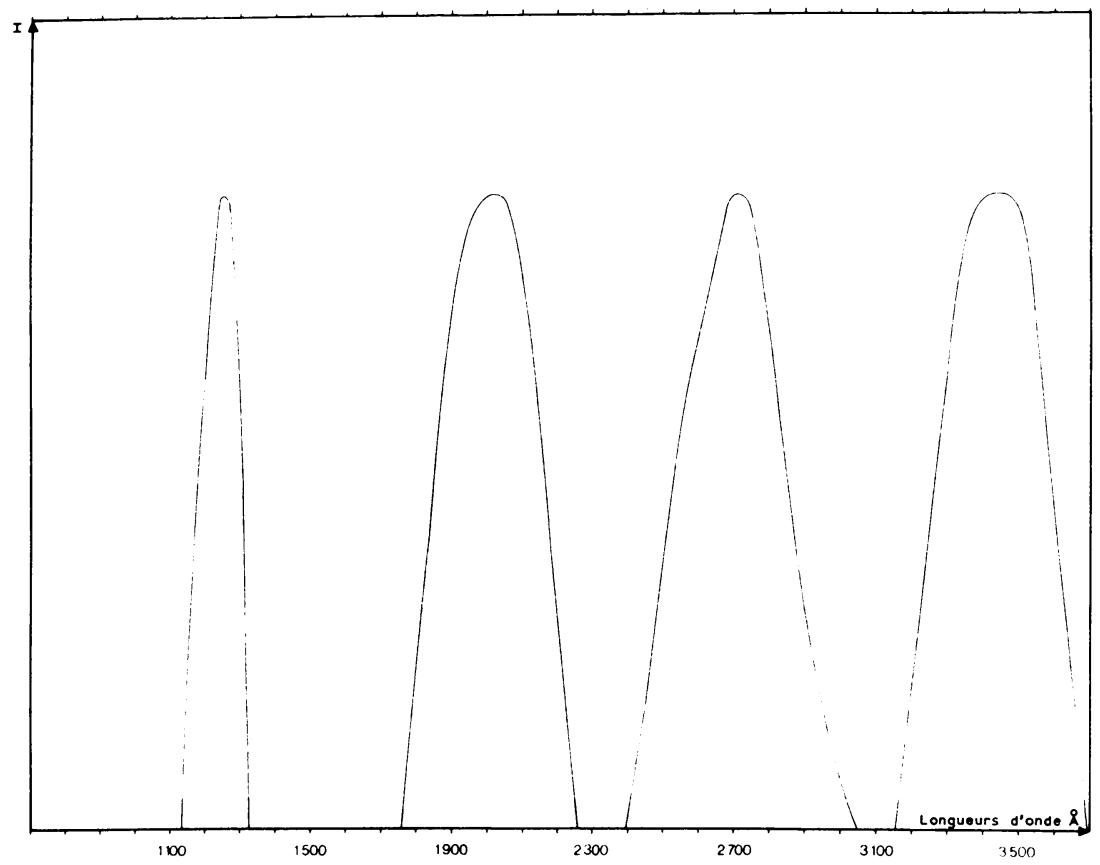

Fig. 2.

On peut donc considérer que la luminance du fond du ciel à $2750 \AA$ est pratiquement indépendante du fond d'étoiles et est essentiellement représentative de la diffusion des poussières interplanétaires. Le fond du ciel peut donc être assimilé à une diffusion en $\lambda^{-1}$ du spectre solaire. Ce résultat est en bon accord avec ceux obtenus par l'OAO II Expérience 'Wisconsin' (Bless, 1969).

L'indice de couleur $U_{2750}^{\prime}-U_{3450}$ a pu être déterminé et l'on trouve que les régions centrales de la nébuleuse d'Andromède sont 3 fois plus brillantes en ultraviolet que l'extrapolation à $2750 \AA$ du spectre visible global du noyau de la nébuleuse.

Ce résultat très intéressant est à rapprocher d'un autre résultat obtenu sur le grand nuage de Magellan (Cruvellier, 1967), puis sur M 33 en 1967 (Carranza et al., 1967. 1968), et depuis, sur plusieurs autres galaxies du système local. 


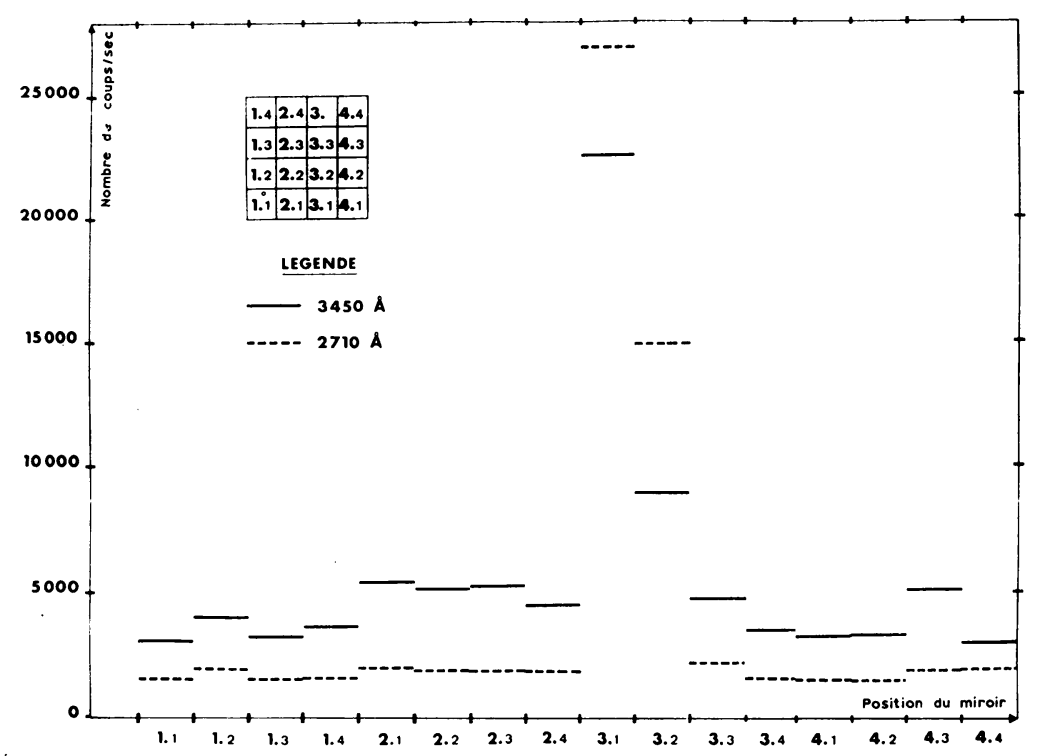

Fig. 3.

Dans les régions centrales de ces nébuleuses, il a été mis en évidence pour la première fois l'émission $\mathrm{H} \alpha$ générale qui indique la présence d'hydrogène ionisé. L'interprétation la plus simple du mécanisme d'excitation est vraisemblablement la présence d'étoiles $\mathrm{B}$ dont le taux dans la population des régions centrales de certaines galaxies (le plus souvent des $\mathrm{Sb}$ ) a certainement été sousestimé. C'est l'interprétation que nous avons retenue pour expliquer l'intensité ultraviolette anormale de M 31 et c'est également cette interprétation que donne dans un article récent Goldberg (1969).

\section{Références}

Bless, R. C.: 1969, Communication privée.

Bonnet, R. M. et Courtès, G.: 1962, Ann. Astrophys. 25, 367.

Carranza, G., Courtès, G., Georgelin, Y., et Monnet, G.: 1967, C. r. hebd. Acad. Sci. Paris 264, 191. Carranza, G., Courtès, G., Georgelin, Y., Monnet, G., et Pourcelot, A.: 1968, Ann. Astrophys. 31, 63. Courtès, G.: 1962, C. r. hebd. Acad. Sci. Paris 254, 1738.

Cruvellier, P.: 1967, Ann. Astrophys. 30, 1059.

Goldberg, L.: 1969, Scient. Amer., juin. 